\title{
Prevalência de medicamentos potencialmente inapropriados para idosos entre os padronizados no Hospital Universitário Regional de Maringá de acordo com os critérios de Beers-Fick
}

\author{
Prevalence of potentially inappropriate medicines for elderly \\ among the standardized ones in the Regional University \\ Hospital of Maringá according to the Beers-Fick rules
}

\section{ABSTRACT}

In Brazil, the demographic transition from young to old country was one of the fastest in the world. The metabolism of drugs in elderly individuals may be changed by various factors that are complicated by the presence of chronic diseases and eventually by acute pathological processes. This usually leads the elderly undergoing pharmacological treatment with many drugs. Some methods can be used to assess the pharmacological treatment of these people such as the Beers-Fick rules, which aims to identify potentially inappropriate medicines for elderly (PIM). The use of the PIM associated with many pharmacological therapy agents increases the incidence of adverse effects. In that way, the purpose of this study is to determine the prevalence of pharmaceutical products potentially inappropriate for the elderly, according to the Beers-Fick rules, in the Regional University Hospital of Maringá. We observed a high prevalence of these medicines (24\%), containing several associated drugs with more than one type of side effects. The results suggest that although the Beers-Fick rules did not include certain drugs subject to adverse reactions, it has been helpful to identify most of these effects. The identification of these adverse reactions allows optimizing drug therapy and reducing health care costs, besides improving the quality of life of elderly patients.

Key Words: inappropriate medications, elderly, adverse effects, Beers-Fick rules

\section{RESUMO}

No Brasil, a transição demográfica de país jovem para idoso foi uma das mais rápidas do mundo. O metabolismo de fármacos em um indivíduo idoso pode ser alterado por vários fatores, sendo agravado pela presença de enfermidades crônicas e eventualmente por processos patológicos agudos. Isso geralmente leva os idosos a serem submetidos à terapêutica farmacológica com diversos fármacos. Alguns métodos podem ser utilizados para se avaliar a terapêutica farmacológica nessas pessoas, como os critérios de Beers-Fick, que pretende identificar medicamentos potencialmente inapropriados para idosos (MPI). O uso de MPI associado à terapêutica farmacológica com diversos fármacos aumenta a incidência de efeitos adversos. Nesse sentido, o propósito deste estudo é verificar a prevalência de MPI para idosos, de acordo com os critérios de Beers-Fick, entre os padronizados no Hospital Universitário Regional de Maringá. Foi observada uma alta prevalência desses medicamentos (24\%), com vários fármacos associados a mais de um tipo de reação adversa. Os resultados sugerem que apesar de os critérios de Beers-Fick não contemplarem alguns fármacos passíveis de reações adversas, ele tem sido útil para identificar a maioria desses efeitos. A identificação dessas reações adversas permite aperfeiçoar a terapêutica farmacológica e diminuir os gastos com a saúde, além de melhorar a qualidade de vida dos pacientes idosos.

Palavras-Chave: fármacos inapropriados, idosos, efeitos adversos, critérios de Beers-Fick 


\section{INTRODUÇÃO}

O Brasil é resultado de transformações, em aspectos da composição etária da população e no perfil epidemiológico. No século XX, as doenças infectocontagiosas eram responsáveis pela metade das mortes registradas no país, hoje correspondendo a menos de $10 \%$, sendo as doenças cardiovasculares a principal causa de morte atualmente. Essas transformações mostram que o Brasil passou de um perfil característico de uma população jovem para outro próprio de faixas etárias mais avançadas, com enfermidades mais complexas e onerosas. A principal causa dessa alteração no padrão demográfico foi a diminuição das taxas de mortalidade, não acompanhada pelos níveis de natalidade. E sse processo se iniciou em 1940, acentuando-se após 1960, devido a uma queda nas taxas de fecundidade, sendo considerada uma das transições demográficas mais rápidas do mundo (1).

Entre as causas dessa redução nas taxas de mortalidade, pode-se citar o aprimoramento do sistema de saúde pública, da previdência social, da infraestrutura urbana e da regulamentação do trabalho, juntamente com o desenvolvimento da indústria químico-farmacêutica (1).

O contínuo aumento da população idosa no mundo todo faz com que seja necessário investir-se na informação e análise do envelhecimento demográfico. Isso possibilita aos administradores públicos definir, formular e avaliar metas e programas direcionados para grupos populacionais específicos, como o dos idosos (2).

De acordo com estimativas do Instituto Brasileiro de Geografia e Estatística (IBGE), a população de indivíduos com 60 anos ou mais irá duplicar no período de 2000 a 2020, passando de 13,9 para 28,3 milhões de idosos. Em 2050 a previsão é de que esse número aumente para 64 milhões (1).Em uma população com prevalência de idosos, as doenças crônicas são as mais comuns, as internações são mais frequentes e o tempo de ocupação do leito é maior, devido à multiplicidade e à natureza das patologias. Esses aspectos do envelhecimento produzem um custo financeiro maior de internação no Sistema Único de Saúde (SUS) (1).

Durante o processo de envelhecimento, o organismo sofre mudanças anatômicas e fisiológicas, podendo ser causadas por alterações naturais e/ou patológicas (3). O metabolismo de fármacos em uma pessoa idosa é alterado devido a vários fatores e, por isso, a terapêutica nesses indivíduos merece atenção especial. Entre os fatores, podemos citar a diminuição da quantidade de água corpórea e aumento da porção lipídica (3). Quando não há ajuste de dose, essas alterações podem levar a uma redução do volume de distribuição de fármacos hidrossolúveis (como lítio e digoxina), resultando em concentrações mais elevadas e predispondo a ocorrência de efeitos adversos. Em contrapartida, fármacos lipossolúveis (como benzodiazepínicos de ação prolongada) têm seu volume de distribuição aumentado, prolongando o tempo para necessário para o efeito máximo e podendo ocorrer acúmulo do medicamento no organismo (4).

Outros fatores que podem alterar a farmacocinética dos medicamentos em indivíduos idosos são: alteração da secreção e esvaziamento gástrico, da motilidade intestinal, do número e capacidade de absorção dos enterócitos, diminuição do fluxo sanguíneo esplâncnico e da concentração plasmática de albumina. Associados a esses fatores, temos ainda como agravantes a presença de variados processos patológicos crônicos, que eventualmente são agravados por processos patológicos agudos, fazendo com que seja necessário se estabelecer uma terapêutica farmacológica com muitos fármacos. Todas essas condições predispõem o paciente idoso a uma maior probabilidade de ocorrer interações farmacológicas e efeitos adversos (5). A terapêutica farmacológica com diversos fármacos é um dos principais fatores de risco para a ocorrência de reações adversas (6).

A excreção de fármacos pode ser realizada por várias vias, no entanto, na grande maioria das vezes ela é efetuada pelos rins. A filtração glomerular pode estar reduzida em $35 \%$ a $50 \%$ entre os 20 e 90 anos de idade (5). As alterações fisiológicas devido à senescência, aliadas a eventuais sobrecargas funcionais nos rins, como por exemplo, alterações volêmicas ou uso de anti-inflamatórios não esteroidais, podem levar o idoso a um quadro de insuficiência renal (3). Dessa forma, pode ocorrer o acúmulo de fármacos e eventuais efeitos tóxicos, se forem mantidas as doses habitualmente utilizadas nos esquemas terapêuticos de idosos (5).

De acordo com Onder et al. (2003), cerca de 3\% das admissões hospitalares de idosos são causadas por eventos adversos. Nesse estudo, os principais tipos de reações encontradas foram: queixas gastrintestinais e complicações metabólicas e hemorrágicas, sendo que o número de fármacos utilizados pelo paciente foi relacionado como o fator de risco mais importante. Levando em conta apenas os eventos adversos graves, outros fatores predisponentes como a idade e o grau de degeneração de vários órgãos foram considerados significativos (7).

O conceito de reação adversa, de acordo com a Organização Mundial de Saúde (OMS), é: qualquer efeito nocivo, não intencional ou indesejado de um fármaco, que ocorre com doses normalmente utilizadas em humanos, para profilaxia, diagnóstico ou terapêutica (8). Lazarou et al. (1998), realizaram um estudo prospectivo para se determinar a ocorrência de efeitos adversos em pacientes hospitalizados (9). A incidência encontrada foi de $6,7 \%$ para os efeitos adversos graves, e de $0,32 \%$ para efeitos adversos fatais. Situações como falhas tera- 
pêuticas, overdose, abuso de fármacos, erros de administração, não concordância e possíveis efeitos adversos foram excluídas desse estudo, o que evidencia que os dados, apesar de serem significativos, não foram superestimados.

De acordo com Wiffen et al. (2002), os fatores associados com o aumento na incidência de efeitos adversos são a idade avançada (especialmente maiores de 70 anos), elevado número de medicamentos, e determinadas classes farmacológicas. As principais classes farmacológicas envolvidas (60-70\%) foram: antibióticos, anticoagulantes, digitálicos, diuréticos, agentes hipoglicemiantes, agentes antineoplásicos e anti-inflamatórios não esteroidais (10). A grande maioria das reações adversas identificadas pertencem ao grupo do tipo A, que são aquelas relacionadas com propriedades conhecidas do fármaco (11); portanto, são previsíveis e potencialmente evitáveis (12).

A ocorrência de efeitos adversos devido à terapêutica com vários fármacos e prescrição de medicamentos inapropriados aumenta os custos financeiros no Sistema de Saúde, fato que poderia ser evitado avaliando-se previamente a terapêutica farmacológica (13).

$\mathrm{O}$ termo "prescrição inadequada" pode abranger várias situações, como: uso de medicamentos que apresentam um alto risco de efeito adverso quando há outra terapia eficaz para a mesma doença e com menor risco, prescrição do medicamento em uma frequência e/ou período maior que o indicado, utilização de medicamentos com conhecida interação fármaco-fármaco ou fármaco-doença e a subutilização de medicamentos clinicamente indicados mas que não são prescritos (4).

Bushardt et al. (2008), observaram que muitos idosos possuem uma terapêutica com diversos fármacos e/ou medicamentos inapropriados. De acordo com os autores: 29,4\% fazem uso de 6 ou mais medicamentos e $15,7 \%$ usam 1 ou mais medicamentos inapropriados (14). O uso de medicamentos inapropriados em idosos é um fator que aumenta consideravelmente a probabilidade de ocorrência de efeitos adversos $(15,16)$.

De acordo com a literatura, há vários instrumentos utilizados para se avaliar e aperfeiçoar a terapêutica farmacológica em pacientes idosos, porém, a maioria desses instrumentos (como testes de fluência verbal, estado mental, nutritivo, motor, de limitação para as atividades funcionais e escalas de depressão geriátrica), envolve a área médica. Poucos envolvem a análise da terapêutica farmacológica e, quando existem, não são utilizados com muita frequência no Brasil (17).

Alguns estudos vêm sendo desenvolvidos com o objetivo de identificar os medicamentos potencialmente inapropriados (MPI) para uso em idosos, seja pelo alto risco de ocorrerem efeitos adversos ou mesmo por falha na eficácia terapêutica (18). Dentre esses estudos, o mais utilizado é o critério de Beers-Fick (19). Esse critério foi criado em 1991, utilizando-se o método de Delphi. O questionário utilizado para a elaboração dos critérios foi aplicado a um grupo de 13 renomados especialistas dos Estados Unidos. Foi alcançado um consenso sobre os critérios a serem utilizados, identificando os MPI para o uso em idosos residentes em casas de repouso (20).

Em 1997, esses critérios foram reavaliados com os seguintes objetivos: incluir novos medicamentos e informações disponíveis na literatura, tornar o método aplicável não apenas aos idosos residentes em casas de repouso, mas também a todos os idosos, independente do seu grau de fragilidade ou de seu local de residência, e atribuir níveis de gravidade aos critérios, além de alertas que levam em conta o diagnóstico do paciente (21).

Em 2003, houve uma nova avaliação de acordo com informações mais recentes disponíveis na literatura científica, excluindo-se ou acrescentando-se novos medicamentos e condições diagnósticas que mereçam maior atenção (22).

Dentro deste contexto, o propósito deste trabalho é avaliar a prevalência de MPI para o uso em idosos, de acordo com os critérios de Beers-Fick, entre os medicamentos padronizados no Hospital Universitário Regional de Maringá

\section{MATERIAL E MÉTODOS}

Os medicamentos padronizados no Hospital Universitário Regional de Maringá (HURM) foram analisados de acordo com os critérios revisados de Beers-Fick. Esses critérios consistem em duas tabelas que listam os medicamentos ou classes de medicamentos inapropriados para idosos. Uma tabela leva em conta o diagnóstico do paciente e a outra, não. Ainda constam informações sobre as reações adversas que podem ocorrer, e o nivel de gravidade delas (22). A lista dos medicamentos padronizados foi cedida por um farmacêtico supervisor, após a aprovação pelo Centro de Assessoria TécnicaCientifica da unidade.

\section{RESULTADOS E DISCUSSÃO}

Nesse estudo foram excluídos medicamentos de uso externo (nasal, dermatológico, otológico, retal e vaginal) por causa da baixa absorção sistêmica, além de formulações com o mesmo princípio ativo, soluções para diálise peritoneal e emulsões para terapêutica nutritiva. Medicamentos que não têm indicação para idosos, como os de uso pediátrico ou em gestantes, também foram excluídos. 
Tabela 1: Medicamentos potencialmente inapropriados, independentemente do diagnóstico ou condição clínica, para uso em idosos no Hospital Universitário de Maringá

\begin{tabular}{|c|c|c|}
\hline MEDICAMENTO & PREOCUPAÇÕES & $\begin{array}{l}\text { NÍVEL DE } \\
\text { GRAVIDADE }\end{array}$ \\
\hline Indometacina & Eventos adversos no sistema nervoso central (SNC). & Alto \\
\hline Amitriptilina (Cloridrato) & Fortes propriedades anticolinérgicas e de sedação. & Alto \\
\hline Lorazepam (doses $>3 \mathrm{mg}$ ) & $\begin{array}{l}\text { Por causa da sensibilidade dos idosos aos benzodiazepínicos, doses menores } \\
\text { podem ser eficazes e seguras. }\end{array}$ & Alto \\
\hline Diazepam & $\begin{array}{l}\text { Longo tempo de meia vida em idosos, produzindo sedação prolongada e } \\
\text { aumentando o risco de quedas e fraturas. Benzodiazepínicos de ação curta e } \\
\text { intermediária são preferíveis. }\end{array}$ & Alto \\
\hline $\begin{array}{l}\text { Digoxina (a dose não deve exce- } \\
\text { der } 0,125 \mathrm{mg} / \text { dia, exceto no tra- } \\
\text { tamento de arritmias atriais) }\end{array}$ & 0 clearence renal diminuído pode aumentar o risco de efeitos adversos. & Baixo \\
\hline Metildopa & Pode causar bradicardia e exacerbar depressão. & Alto \\
\hline Atropina (Sulfato) & $\begin{array}{l}\text { Fármacos anti-espasmódicos gastrintestinais são anticolinérgicos e têm efe- } \\
\text { tividade incerta. Essas drogas devem ser evitadas (especialmente no uso a } \\
\text { longo prazo). }\end{array}$ & Baixo \\
\hline $\begin{array}{l}\text { Prometazina, Dexclorfeniramina } \\
\text { (Maleato) }\end{array}$ & $\begin{array}{l}\text { Anti-histamínicos que podem ter potente atividade anticolinérgica. Anti- } \\
\text { histamínicos sem ação colinérgica são preferíveis. }\end{array}$ & Alto \\
\hline Sulfato Ferroso (>325mg/dia) & $\begin{array}{l}\text { Dosagens acima desta não aumentam a absorção, mas aumentam a incidên- } \\
\text { cia de constipação. }\end{array}$ & Baixo \\
\hline $\begin{array}{l}\text { Tiopental Sódico (exceto quando } \\
\text { usado para controlar convulsões) }\end{array}$ & $\begin{array}{l}\text { Pode causar dependência.É mais propenso a causar efeitos adversos do que } \\
\text { outros sedativos hipnóticos. }\end{array}$ & Alto \\
\hline Petidina/Meperidina (Cloridrato) & $\begin{array}{l}\text { Não é um analgésico eficiente em doses comuns. Pode causar confusão e tem } \\
\text { várias desvantagens com relação a outras drogas narcóticas. }\end{array}$ & Alto \\
\hline Fluoxetina (Cloridrato) & $\begin{array}{l}\text { Longo tempo de meia vida, risco de causar estimulação excessiva do SNC, per- } \\
\text { turbações do sono e agitação. Alternativas mais seguras estão disponíveis. }\end{array}$ & Alto \\
\hline $\begin{array}{l}\text { Bisacodil (uso a longo prazo), } \\
\text { exceto quando há uso de analgé- } \\
\text { sicos opióides }\end{array}$ & Pode ocasionar disfunção intestinal. & Alto \\
\hline Amiodarona (Cloridrato) & $\begin{array}{l}\text { Associada com problemas no intervalo QT do eletrocardiograma, risco de } \\
\text { causar torsade de pointes, falta de eficácia em idosos. }\end{array}$ & Alto \\
\hline Isoxsuprina (Cloridrato) & Falta de eficácia. & Baixo \\
\hline Nitrofurantoína & $\begin{array}{l}\text { Potencial para causar insuficiência renal. Alternativas mais seguras estão } \\
\text { disponiveis. }\end{array}$ & Alto \\
\hline Doxazosina (Mesilato) & Potencial para causar hipotensão, boca seca e problemas urinários. & Baixo \\
\hline Clonidina (Cloridrato) & Potencial para hipotensão ortostática e efeitos adversos no SNC. & Baixo \\
\hline Óleo Mineral & $\begin{array}{l}\text { Pode ocorrer aspiração e efeitos adversos. Alternativas mais seguras estão } \\
\text { disponíveis. }\end{array}$ & Alto \\
\hline
\end{tabular}

adaptado de Fick et al. (2003) (22).

Foram encontrados 61 MPI para idosos entre os 253 medicamentos padronizados no Hospital Universitário Regional de Maringá (aproximadamente $24 \%$ ). Esses medicamentos podem ser identificados nas Tabelas 1 e 2. Pode ser observado que muitos desses MPI, como benzodiazepínicos, alguns antidepressivos e relaxantes musculares, estão envolvidos em mais de um tipo de reação adversa, o que evidencia que devem ser utilizados com cuidado em faixas etárias mais avançadas. 
Tabela 2: Medicamentos potencialmente inapropriados, considerando o diagnóstico ou condição clínica, para uso em idosos no Hospital Universitário de Maringá

\begin{tabular}{|c|c|c|c|}
\hline Doença ou condição & Medicamento & Preocupações & $\begin{array}{l}\text { Nivel de } \\
\text { severidade }\end{array}$ \\
\hline Insuficiência Cardíaca & $\begin{array}{l}\text { Bicarbonato de sódio, Cloreto de sódio, } \\
\text { Ringer lactato, Reidratante oral, Hidro- } \\
\text { xietilamido/Cloreto de sódio, Solução } \\
\text { glicofisiológica }\end{array}$ & $\begin{array}{l}\text { Efeito inotrópico negativo. Pode promo- } \\
\text { ver retenção de fluidos e exacerbar a in- } \\
\text { suficiência cardíaca. }\end{array}$ & Alto \\
\hline Hipertensão & Efedrina (sulfato) & $\begin{array}{l}\text { Pode produzir elevação na pressão arterial } \\
\text { devido à atividade simpatomimética. }\end{array}$ & Alto \\
\hline $\begin{array}{l}\text { Úlceras gástricas ou } \\
\text { duodenais }\end{array}$ & $\begin{array}{l}\text { Ácido acetilsalicílico (>325mg), Diclofe- } \\
\text { naco, Paracetamol/Codeína, Ibuprofeno, } \\
\text { Paracetamol, Tenoxicam, Indometacina }\end{array}$ & $\begin{array}{l}\text { Podem exacerbar úlceras pré-existentes } \\
\text { ou induzir a formação delas. }\end{array}$ & Alto \\
\hline Convulsões ou epilepsia & Clorpromazina & Pode diminuir o limiar das convulsões. & Alto \\
\hline $\begin{array}{l}\text { Distúrbios da } \\
\text { coagulação do sangue } \\
\text { ou terapia com } \\
\text { anticoagulantes }\end{array}$ & $\begin{array}{l}\text { Ácido acetilsalicílico, Diclofenaco, Ibu- } \\
\text { profeno, Indometacina, Paracetamol, } \\
\text { Paracetamol/Codeína, Tenoxicam, Clopi- } \\
\text { dogrel }\end{array}$ & $\begin{array}{l}\text { Pode prolongar o tempo de coagulação e } \\
\text { elevar os valores de INR (Razão Normali- } \\
\text { zada Internacional), ou inibir a agregação } \\
\text { plaquetária, resultando em um aumento } \\
\text { do potencial de sangramento. }\end{array}$ & Alto \\
\hline $\begin{array}{l}\text { Obstrução do fluxo } \\
\text { urinário }\end{array}$ & $\begin{array}{l}\text { Dexclorfeniramina (maleato), Dimenidri- } \\
\text { nato/Piridoxina (cloridrato), Prometazina } \\
\text { (cloridrato), Atracúrio (besilato), Rocurô- } \\
\text { nio (brometo), Baclofeno, Pancurônio, } \\
\text { Suxametônio (cloreto), Biperideno, Esco- } \\
\text { polamina (Butilbrometo), Escopolamina } \\
\text { (butilbrometo)/Dipirona sódica, Ipratró- } \\
\text { pio (Brometo), Atropina (sulfato), Ami- } \\
\text { triptilina (cloridrato), Fluoxetina (clori- } \\
\text { drato), Imipramina (cloridrato) }\end{array}$ & $\begin{array}{l}\text { Pode diminuir o fluxo de urina, levando à } \\
\text { retenção urinária. }\end{array}$ & Alto \\
\hline $\begin{array}{l}\text { Incontinência urinária } \\
\text { de esforço }\end{array}$ & $\begin{array}{l}\text { Doxazosina (mesilato), Imipramina (clo- } \\
\text { ridrato), Amitriptilina (cloridrato), Bi- } \\
\text { perideno, Escopolamina (Butilbrometo), } \\
\text { Escopolamina (butilbrometo)/ Dipirona } \\
\text { sódica, Ipratrópio (Brometo), Atropina } \\
\text { (sulfato), Diazepam }\end{array}$ & $\begin{array}{l}\text { Pode ocasionar poliúria e piora da incon- } \\
\text { tinência }\end{array}$ & Alto \\
\hline Arritmias & $\begin{array}{l}\text { Imipramina (cloridrato), Amitriptilina } \\
\text { (cloridrato) }\end{array}$ & $\begin{array}{l}\text { Efeitos pró-arrítmicos e capacidade para } \\
\text { produzir alterações no intervalo QT do } \\
\text { eletrocardiograma. }\end{array}$ & Alto \\
\hline Doença de Parkinson & $\begin{array}{l}\text { Metoclopramida (Cloridrato), Clorpro- } \\
\text { mazina, Levomepromazina, Haloperidol, } \\
\text { Droperidol e Fentanila/Droperidol }\end{array}$ & Efeitos antidopaminérgicos/colinérgicos. & Alto \\
\hline Transtornos cognitivos & $\begin{array}{l}\text { Fenobarbital, Tiopental sódico, Bipe- } \\
\text { rideno, Escopolamina (butilbrometo), } \\
\text { Escopolamina (butilbrometo)/Dipirona } \\
\text { sódica, Ipratrópio (brometo), Atropina } \\
\text { (sulfato), Baclofeno, Atracúrio (besi- } \\
\text { lato), Rocurônio (Brometo), Pancurônio, } \\
\text { Suxametônio (cloreto) }\end{array}$ & Efeitos que alteram funções do SNC. & Alto \\
\hline Depressão & $\begin{array}{l}\text { Metildopa e uso a longo prazo de: Cloba- } \\
\text { zam, Clonazepam, Diazepam, Lorazepam } \\
\text { e Midazolam. }\end{array}$ & Pode produzir exacerbação da depressão. & Alto \\
\hline Anorexia e má nutrição & Fluoxetina (cloridrato) & Efeitos supressores do apetite. & Alto \\
\hline
\end{tabular}


Tabela 2 (cont): Medicamentos potencialmente inapropriados, considerando o diagnóstico ou condição clínica, para uso em idosos no Hospital Universitário de Maringá

\begin{tabular}{|c|c|c|c|}
\hline Doença ou condição & Medicamento & Preocupações & $\begin{array}{l}\text { Nivel de } \\
\text { severidade }\end{array}$ \\
\hline Síncope e quedas & $\begin{array}{l}\text { Clonazepam, Clobazam, Lorazepam, Mida- } \\
\text { zolam, Imipramina (cloridrato), Amitrip- } \\
\text { tilina (cloridrato) }\end{array}$ & $\begin{array}{l}\text { Pode produzir ataxia, alteração da função } \\
\text { psicomotora, quedas e síncopes. }\end{array}$ & Alto \\
\hline $\begin{array}{l}\text { Síndrome da secreção } \\
\text { inadequada do } \\
\text { hormônio anti- } \\
\text { diurético }\end{array}$ & Fluoxetina (cloridrato) & Pode exacerbar ou causar a síndrome. & Baixo \\
\hline $\begin{array}{l}\text { Doença pulmonar } \\
\text { obstrutiva crônica }\end{array}$ & Diazepam, Propranolol (cloridrato) & $\begin{array}{l}\text { Efeitos adversos no SNC. Pode induzir ou } \\
\text { causar depressão respiratória. }\end{array}$ & Alto \\
\hline Constipação crônica & $\begin{array}{l}\text { Anlodipino (besilato), Diltiazem (clo- } \\
\text { ridrato), Nifedipino, Nimodipino, Vera- } \\
\text { pamil (cloridrato), Biperideno, Atropina } \\
\text { (sulfato), Escopolamina (Butilbrometo), } \\
\text { Escopolamina (butilbrometo)/Dipirona } \\
\text { sódica, Ipratrópio (brometo), Imipramina } \\
\text { (cloridrato), Amitriptilina (cloridrato) }\end{array}$ & Pode exacerbar a constipação. & Baixo \\
\hline
\end{tabular}

adaptado de Fick et al. (2003) (22).

\section{CONCLUSÃO}

Os resultados desse estudo sugerem uma alta prevalência de medicamentos inapropriados para idosos entre os fármacos padronizados no Hospital Universitário Regional de Maringá. Além disso, vários desses medicamentos estão envolvidos em mais de um tipo de possível reação adversa. Os critérios de Beers-Fick, apesar de não contemplarem a análise de alguns fármacos passí- veis de reações adversas, podem ser úteis para se avaliar a terapêutica farmacológica em indivíduos idosos e para identificar possíveis reações adversas provocadas por determinados tipos de medicamentos. Por meio desse instrumento, é possível melhorar a qualidade de vida dos idosos, além de diminuir os custos financeiros com a saúde.

\section{REFERÊNCIAS}

1. IBGE. Instituto Brasileiro de Geografia e Bioestatística. Indicadores sociodemográficos e de saúde no Brasil. n. 25. Rio de Janeiro; 2009.

2. UN. United Nations. World Population Ageing: 2009. New York; 2010.

3. Jacob FW, Souza RR. Anatomia e fisiologia do envelhecimento. In: Carvalho ETF, Netto MP. Geriatria: Fundamentos, Clínica e Terapêutica. 1 ed. São Paulo: Atheneu, 2000, p. 31-40.

4. Gallagher P, Barry P, O'Mahony D. Inappropriate prescribing in the elderly. J Clin Pharm Ther. 2007;32(2):113-21.

5. Carvalho FET, Netto MP, Pasini U. Farmacocinética e Farmacodinâmica das drogas. In: Carvalho, ETF, Netto MP. Geriatria: Fundamentos, Clínica e Terapêutica. 1 ed. São Paulo: Atheneu, 2000, p. 409-421.
6. Hanlon JT, Pieper CF, Hajjar ER, Sloane RJ, Lindblad CI, Ruby CM, Schmader KE. Incidence and Predictors of All and Preventable Adverse Drug Reactions in Frail Elderly Persons After Hospital Stay. J Gerontol A Biol Sci Med Sci. 2006;61(5):511-5.

7. Onder G, Landi F, Cesari M, Gambassi G, Carbonin P, Bernabei R. Inappropriate medication use among hospitalized older adults in Italy: results from the Italian Group of Pharmacoepidemiology in the Elderly. Eur J Clin Pharmacol. 2003;59(2):157-62.

8. WHO. World Health Organization. International Drug Monitoring: The Role of the Hospital. Report of a WHO Meeting (WHO Technical Report Series 425), 1969.

9. Lazarou J, Pomeranz BH, Corey PN. Incidence of adverse drug reactions in hospitalized patients: A meta-analysis of prospective studies. JAMA. 1998;279(15):1200-5. 
10. Wiffen P, Gill M, Edwards J, Moore A. Adverse drug reactions in hospital patients. Bandolier Extra. 2002: 1 - 15.

11. Moore N, Lecointre D, Noble C, Mobile M. Frequency and cost of serious adverse drug reactions in a department of general medicine. Br J Chin Pharmacol. 1998;45(3):301-8.

12. Green CF, Mottram DR, Rowe PH, Pirmohamed M. Adverse drug reactions as a cause of admission to an acute medical assessmont unit: a pilot study. J Chin Pharm There. 2000;25(5):355-61.

13. Cahir C, Fahey T, Feeling M, Teljeur C, Feely J, Bennett K. Potentially inappropriate prescribing and cost outcomes for older people: a national population study. $\mathrm{Br} \mathrm{J}$ Chin Pharmacol. 2010;69(5):543-52.

14. Bushardt RL, Massey EB, Simpson TW, Ariail JC, Simpson KN. Polypharmacy: Misleading, but manageable. Chin Intern Aging. 2008;3(2):383-9.

15. Chrischilles EA, VanGilder R, Wright K, Kelly M, Wallace RB. Inappropriate Medication Use as a Risk Factor for Self-Reported Adverse Drug Effects in Older Adults. J Am Geriatr Soc. 2009;57(6):1000-6.

16. Albert SM, Colombia A, Hanlon J. Potentially Inappropriate Medcations and Risk of Hospitalization in Retirees. Drugs Aging. 2010;27(5):407-15.
17. Quinalha JV, Correr CJ. Instrumentos para avaliação da farmacoterapia do idoso: una revisão. Rev Bras Ger Geront. 2010;13(3):487-500.

18. Nóbrega $\mathrm{O}$ de $\mathrm{T}$, Karnikowski $\mathrm{MG}$ de $\mathrm{O}$. Pharmacotherapy in the elderly: precautions with medication. Ciên Saúde Col. 2005;10(2):309-13.

19. Soars MA, Fernandez-Llimos F, Cabrita J, Morris J. Tools to evaluate potentially inappropriate prescription in the elderly: a systematic review. Acts Med Port. 2011;24(5):775-84.

20. Beers MH, Ouslander JG, Rollingher I, Reuben DB, Brooks J, Beck JC. Explicit criteria for determining inappropriate medication use in nursing home residents. Arch Intern Med. 1991;151(9):1825-32.

21. Beers MH. Explicit criteria for determining potentially inapproprate medication use by the elderly: An update. Arch Intern Med. 1997;157(14):1531-6.

22. Tick DM, Cooper JW, Wade WE, Waller JL, Maclean JR, Beers $\mathrm{MH}$. Updating the Beers criteria for potentially inappropriate medication use in older adults: results of a US consensus panel of experts. Arch Intern Med. 2003;163(22):2716-24.

10 